\title{
Impact of the COVID-19 Pandemic on Colorectal Cancer Screening: a Systematic Review
}

\author{
Afrooz Mazidimoradi $^{1} \cdot$ Azita Tiznobaik $^{2} \cdot$ Hamid Salehiniya $^{3}$
}

Accepted: 24 July 2021 / Published online: 18 August 2021

○) Springer Science+Business Media, LLC, part of Springer Nature 2021

\begin{abstract}
Background After the World Health Organization (WHO) announcing about global pandemic of COVID-19 in March 2020 and relocation of health care resources for controlling this infection, cancer screening programs especially colorectal cancer (CRC) have been suspended in many countries. According to GLOBOCAN 2020 data, CRC is the third prevalent and second deadliest cancer in the world. So, early detection through screening is essential to reduce the mortality associated with this cancer. The present study was designed to investigate the impact of COVID-19 pandemic on colorectal cancer screening.

Methods and Materials A comprehensive search performed on June 2021 in various databases, including Medline, Web of Science, and Scopus. Keywords such as "Early Detection," "Cancer," "Cancer Screening," "Cancer Screening Tests," "Coronavirus Disease-19," "COVID 19," "Coronavirus Disease," "SARS-CoV-2 Infection," "SARS-CoV-2," "2019-nCoV," "coronavirus, 2019 Novel," "SARS COV 2 Virus," "Severe Acute Respiratory Syndrome Coronavirus 2," "COVID-19," "COVID-19, Coronavirus Disease 19," "SARS Coronavirus 2","Colorectal neoplasm” and "Colorectal Cancer" were used individually or in combination to search. All articles were entered into Endnote X7 software that remove duplicates. Then, studies were first selected by title and then by abstract and at the end full texts were investigated.

Results Of the 850 identified studies, 25 were identified as eligible. The results of studies show that in general, colorectal cancer screening has decreased from 28 to $100 \%$ in different countries and at different times after the onset of the COVID-19 pandemic. During this period, only 2 to $2.5 \%$ of hospitals and screening centers with $100 \%$ capacity continued to operate, and more than $77 \%$ of them limited their activities to less than $10 \%$ of their normal capacity. Also, completion of colonoscopies requiring examination showed a decrease of $65.7 \%$, surveillance colonoscopy showed a decrease of 44.6 to $79 \%$, prescription colonoscopy decreased 60 to $81 \%$, and referrals to colonoscopy showed a $43 \%$ decline. However, emergency colonoscopy shows a 2 to $9 \%$ increase. The use of the Fecal immunochemical test (FIT) test is also generally declining but is increasing in areas used as a colonoscopy alternative.

Conclusions Considering that the reduction in colorectal cancer screening following COVID-19 pandemic is due to the restrictions imposed for the high prevalence of COVID-19 disease and the lack of referrals due to the fear of developing COVID-19 infection; compensating for the decline and preventing the continuation of this decreasing trend requires serious and effective interventions to maintain the capacity of screening services during the COVID-19 crisis, increase the capacity of screening centers during the lifting of restrictions and reduce fear in the public.
\end{abstract}

Keywords Screening $\cdot$ COVID-19 $\cdot$ Colorectal cancer $\cdot$ Systematic review

Hamid Salehiniya

alesaleh70@yahoo.com

Shiraz University of Medical Sciences, Shiraz, Iran

2 Department of Midwifery and Reproductive Health, Mother and Child Care Research Center, School of Nursing and Midwifery, Hamadan University of Medical Sciences, Hamadan, Iran

3 Social Determinants of Health Research Center, Birjand University of Medical Sciences, Birjand, Iran

\section{Introduction}

Following the diagnosis of an acute respiratory infectious disease called SARS-COV-2 in early November 2019 in Wuhan, China, and the World Health Organization (WHO) announcing a global pandemic in March 2020 [1], the relocation of staff and health care resources for addressing the pandemic has become a necessity; therefore, cancer 
screening programs for asymptomatic patients have been suspended in many countries [2]. Thus, preventive care services including colorectal cancer (CRC) screening in health systems have seen a dramatic decline worldwide [3].

Primary prevention is the main strategy to reduce the growing global burden of colon cancer [4]; however, it should be noted that colorectal cancer, with a mortality rate of $9.4 \%$, is the second cause of cancer death in the world after lung cancer and the third leading cause of cancer death in men and women. It is also the third most common cancer among men and the second most common cancer in women [5].

More than 1.9 million new cases and 935,000 deaths from this cancer occurred in 2020 [5]; therefore, early detection through routine screening is also necessary to improve the mortality rate of this cancer. Since the dramatic reduction in cancer screening due to the Covid-19 pandemic has had a significant impact on the diagnosis of cancer, especially colon cancer, diagnosis at advanced stages or later stages, delayed onset of treatment, decreased quality of life, and increased mortality are associated problems of the COVID19 pandemic [6].

Yong et al. reported that a 6-month delay in the initial screening of colorectal cancers could increase the incidence of this cancer to 2200 and the death rate to 960 . Even when screening resumes, longer intervals and reduced screening rates increase more cancer deaths [7]. A study in the UK also showed that the diagnosis of colorectal cancer was significantly reduced (62\%) after the onset of the COVID-19 epidemic. It had also the most delay in diagnosis compared to other cancers [8].

Delays in colon cancer surgery have also been shown to be associated with lower survival $[9,10]$. Issaka et al. showed that increasing the use of fecal immunochemical test (FIT) from 15 to $22 \%$ over a 3 -year period to compensate for the decrease in COVID-19 colonoscopy screening was associated with 655,825 more screening and 2715 colon cancer which $72 \%$ were diagnosed in the early stages [11]. Therefore, considering the effects of the COVID-19 epidemic on colorectal cancer screening and the importance of knowing about the reduction in the number of screening on predicting the consequences in health planning, The present study was designed to investigate the impact of COVID-19 pandemic on colorectal cancer screening.

\section{Methods and Materials}

\section{Search Strategy}

This systematic review was conducted in accordance with the Systematic Review Checklist (PRISMA) and by searching for related articles in three databases of PubMed/
MEDLINE, Scopus, and Web of Science, using the following keywords from 2020 to 2021: "Early Detection," "Cancer," "Cancer Screening," "Cancer Screening Tests," "Coronavirus Disease-19," "COVID 19," "Coronavirus Disease," "SARS-CoV-2 Infection," "SARS-CoV-2," "2019-nCoV," "coronavirus, 2019 Novel," "SARS COV 2 Virus," "Severe Acute Respiratory Syndrome Coronavirus 2," "COVID-19," "COVID-19, Coronavirus Disease 19," "SARS Coronavirus 2," "Colorectal neoplasm"and "Colorectal Cancer" The AND, OR, and Mesh terms operators were also used to improve the search result. Also a manual search was performed in reputable scientific journals to find articles related to the full text.

\section{Inclusion and Exclusion Criteria}

All types of observational studies, addressing the impact of COVID-19 pandemic on colorectal cancer screening, published in the English language were included in the review. Studies that did not address the specific effects of coronavirus on cancer screening, or included patients diagnosed with cancer before the epidemic or patients with a symptomatic diagnosis of early cancer, were excluded. Review studies, case reports, letters to editors, commentaries, and reports were also excludded.

\section{Screening and Selection of Studies}

All articles searched in the databases were entered into Endnote $\mathrm{X} 7$ software. After removing duplicates, studies were first selected by title and then by abstract according to the relevance of the titles and abstracts with the purpose of the study. Their eligibility was then verified by reviewing the full text. Articles that evaluated every aspect of colorectal cancer screening during the COVID-19 epidemic were included in the analysis.

\section{Data Extraction}

To extract the data, the prepared checklist was used and the following information was extracted from each study: surname of the first author, year of publication, country of study, type of study, sample size, age and sex of the target group, period of evaluation, and the main findings.

\section{Quality Assessment}

"Adapted Newcastle-Ottawa Quality Assessment Scales" checklist was used to evaluate the quality of the articles in this review. (http://www.ohri.ca/programs/clinical_ epidemiology/oxford.asp). This tool consists of 3 separate sections: selection, comparison, and conclusion. Studies were scored based on overall scores and divided into 3 categories: good, moderate, and poor. 


\section{Result}

\section{Selection of Studies}

The search result in the databases based on the intended keywords included 850 articles, which after deleting duplicates (403 articles), according to the title and abstract of the remaining articles, 382 articles were deleted. Afterward, a thorough review of the remaining articles was performed; then, 14 other articles were excluded due to publication in a language other than English [4 articles], a letter to the editor [ 4 articles], etc. Subsequently, the full text of the articles reviewed, 7 articles were deleted due to lack of access to the full text or inconsistency with the objectives of the study, and finally, 25 articles were analyzed in this systematic review (Fig. 1).

\section{Characteristics of Studies and Quality Assessment}

The eligible articles that were considered for analysis were divided into the following groups: colonoscopy and sigmoidoscopy screening in general [12-28], surveillance colonoscopy [12, 13, 18], prescribed colonoscopy [12, 13, 18, 24], emergency colonoscopy $[12,14]$, non-invasive methods [16, 18], referrals to screening colonoscopy [29], completing of screening colonoscopies that needed to be reviewed [12], responding to recalls $[12,24,30]$, participating in screening program [31], sales of laboratory materials needed for colonoscopy [32], reducing the capacity of screening centers [33, 34 ], changing the screening method and admission process in the screening centers $[34,35]$. Based on the review using the checklist, 22 articles/studies were of good quality and 3 articles/studies were of medium quality. The results are summarized in Table 1.

\section{Study Results}

\section{Colonoscopy and Sigmoidoscopy Screening in General}

In a study by Wassie et al. comparing the number of colonoscopies in Australia in two similar time periods (April-June) in 2019 and 2020, they found that during the period in 2020, the total number of completed colonoscopies decreased by $51.1 \%(n=569)$ compared to the same months in 2019 $(n=1164)$, with the largest decrease of $88 \%$ observed in April 2020, and colonoscopy at the Noarlunga Health Service (NHS) in April 2020 reached to zero [12].

A study by Tinmouth et al. in Canada that compared colonoscopy cases from March to June 2020 with the same time period in 2019 found that total colonoscopy cases decreased by $60 \%$ in 2020 compared to 2019 , from 107,034 cases in 2019 to 36,029 cases in 2020 [13].

Shinkwin et al. in their study in the UK showed that the average number of diagnostic endoscopy cases (colonoscopy and flexible sigmoidoscopy) performed per month for 2020 compared to 2018 and 2019 shows a significant decrease so that the total number of diagnostic colonoscopies performed from February 2020 (coinciding with the beginning of the National restrictions) has significant decline observed that reached a peak in April 2020. Despite the gradual increase in diagnostic endoscopy cases, this number has not yet reached the number observed in 2019 and 2018 so that the average number of diagnostic endoscopy cases in April 2020 compared to April 2018 and 2019 decreased by approximately $28 \%$ [14].

A study by Rutter et al. in the UK compared the activity of endoscopic centers and the diagnosis of cancer in the three periods, before COVID-19 (6 January 2020 to 15 March), transition period (16-22 March), and the period influenced by COVID-19 (23 March-31 May). They found that, compared to the pre-COVID-19 period, the average number of low colonoscopy cases by $90 \%$, flexible sigmoidoscopy by $91 \%$, and esophagogastroduododenoscopy (OGD) by $86 \%$ has decreased per week and the total reduction for all methods was $87.8 \%$. In contrast, this reduction was only $44 \%$ for endoscopic retrograde cholangiopancreatography (ERCP) procedures [15].

Myint et al. in a study conducted in the USA, found that in the period before COVID-19 (1/29/2020-3/17/2020), the average screening test was $382.4 \pm 54.8$; colonoscopy $(222.6 .0 \pm 33.0$ per week) and FIT $(154.0 \pm 25.2$ per week $)$ were most screening tests performed per week, while during the stop period $(3 / 18 / 2020-5 / 4 / 2020)$, the total average decreased to $74.3 \pm 47.1$ per week $(p<0.01)$, as colonoscopy showed the most severe decline $(11.4 \pm 11.1 ; p<0.01)$. They also found that in the months following the resumption of elective endoscopy (5/5/2020-10/27/2020), overall use of the screening test increased significantly equal to the preepidemic rate $(346.8 \% \pm 93.4 ; p=0.53)$. However, it did not correspond to pre-pandemic performance (174.2 \pm 47.1 ; $p<0.02$ ) [16].

In a study by Longcroft-Wheaton et al. in the UK, after comparing the activity of endoscopic centers in 8 weeks of spring, summer, and autumn in 2019 and the first 6 weeks of the COVID-19 crisis, they found that the average colonoscopy declined from 86 cases per week to 12 cases and the average of flexible sigmoidoscopy decreased from 108 cases per week to 10 cases. Also, the endoscopic procedures required to diagnose colorectal cancer have been reduced from 47 to 12 per week [17].

Lantinga et al. in the Netherlands saw a $45 \%$ reduction (from 12,219 to 5609) in colonoscopy cases by comparing 


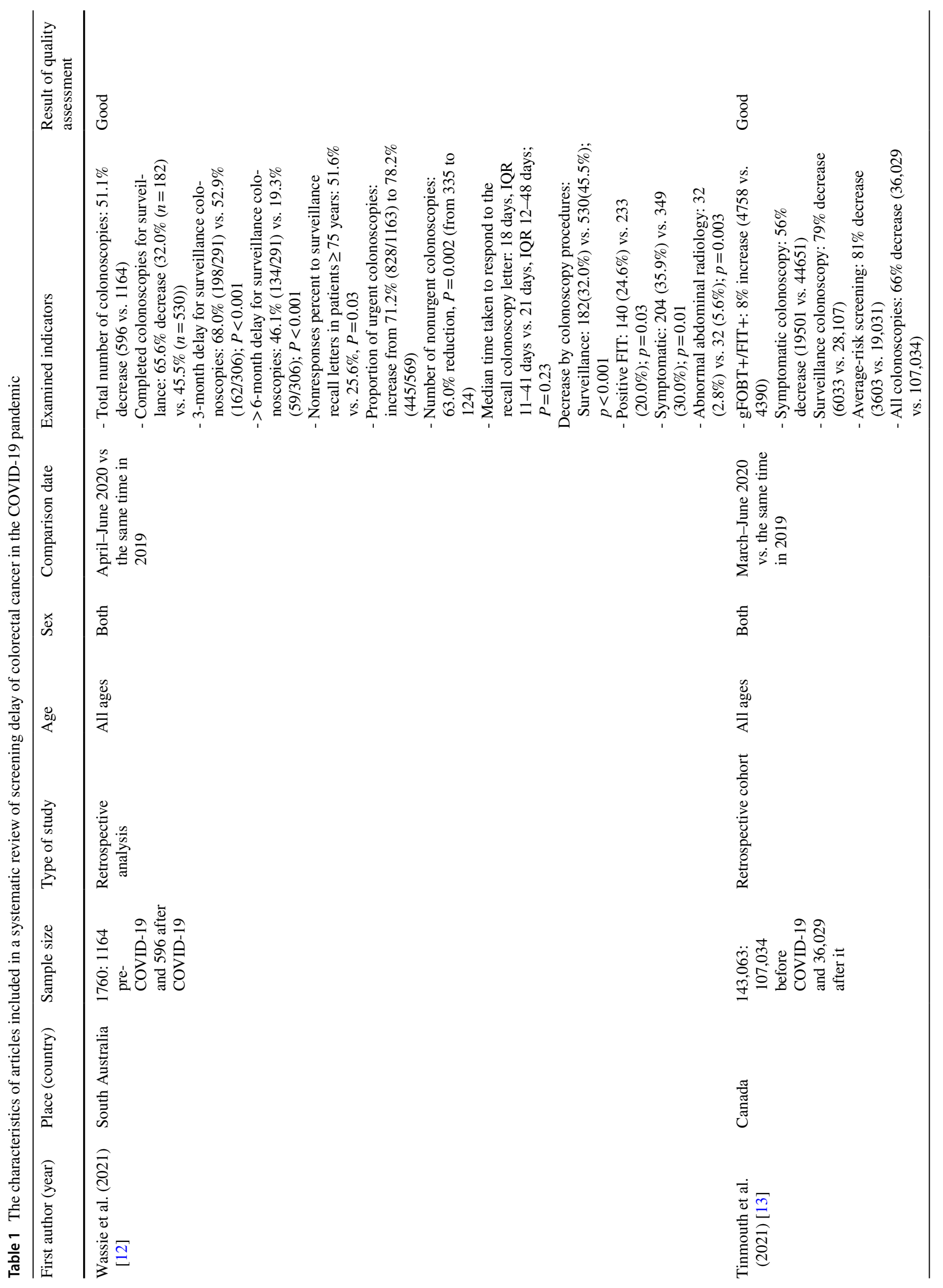




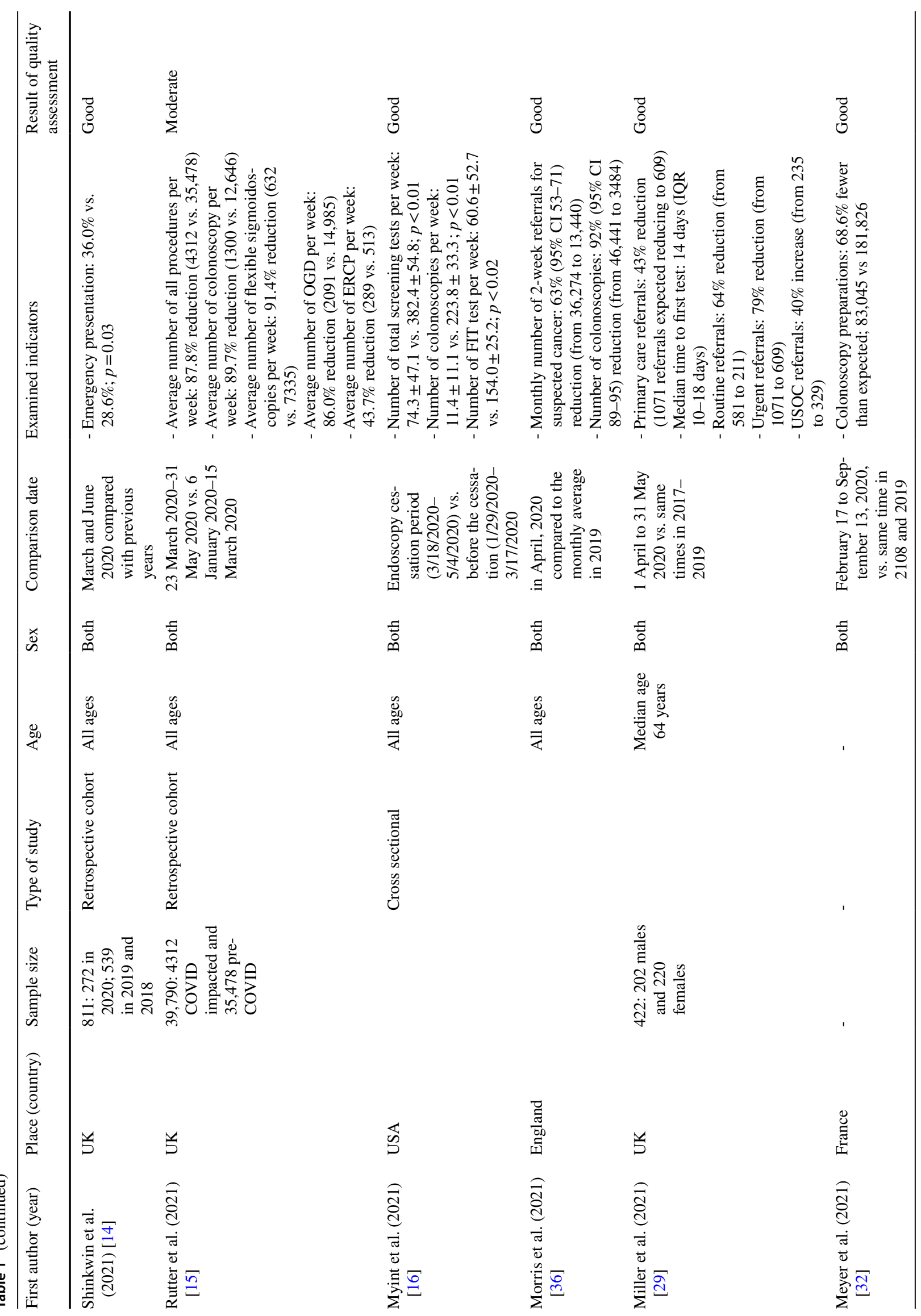




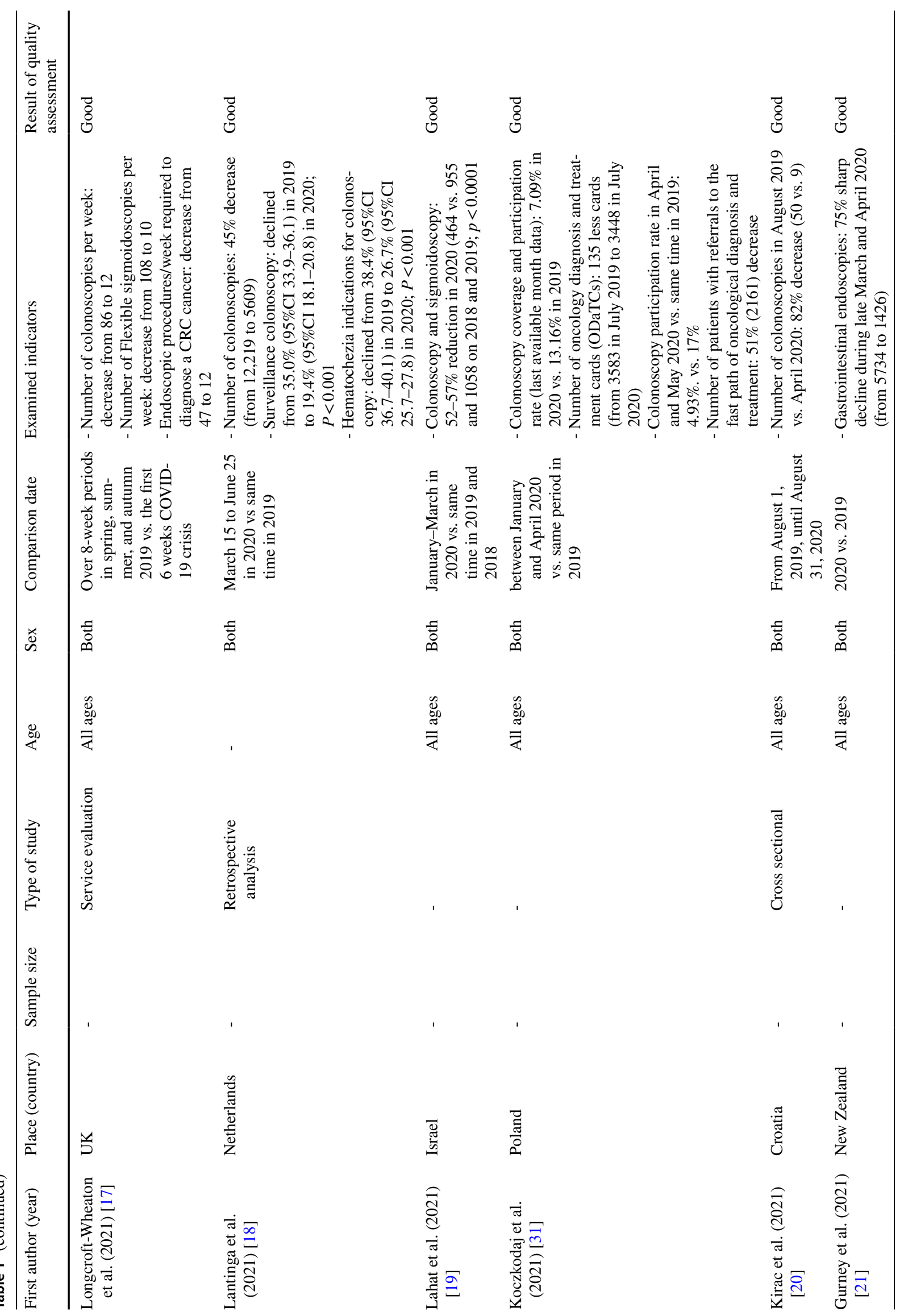




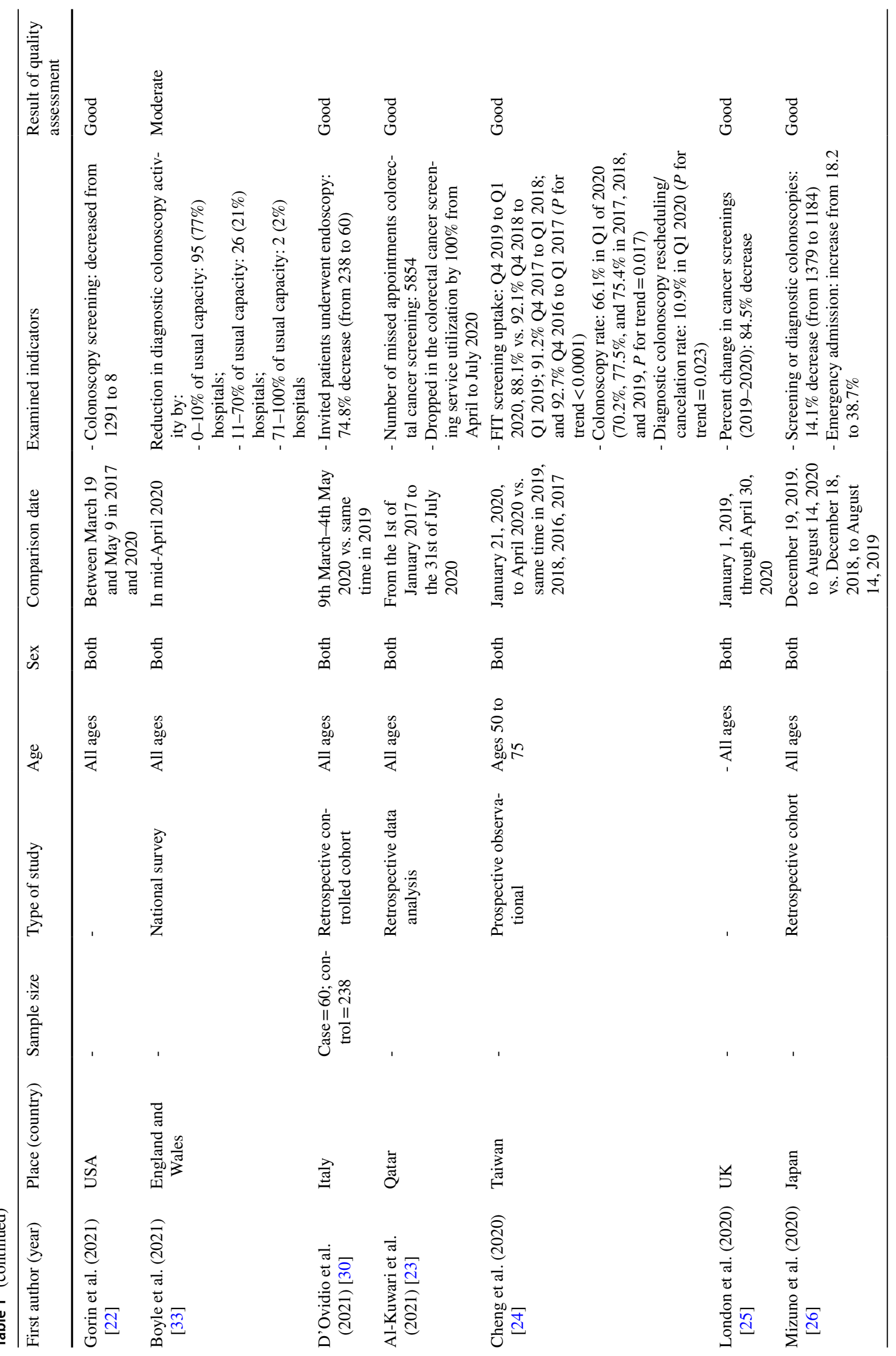




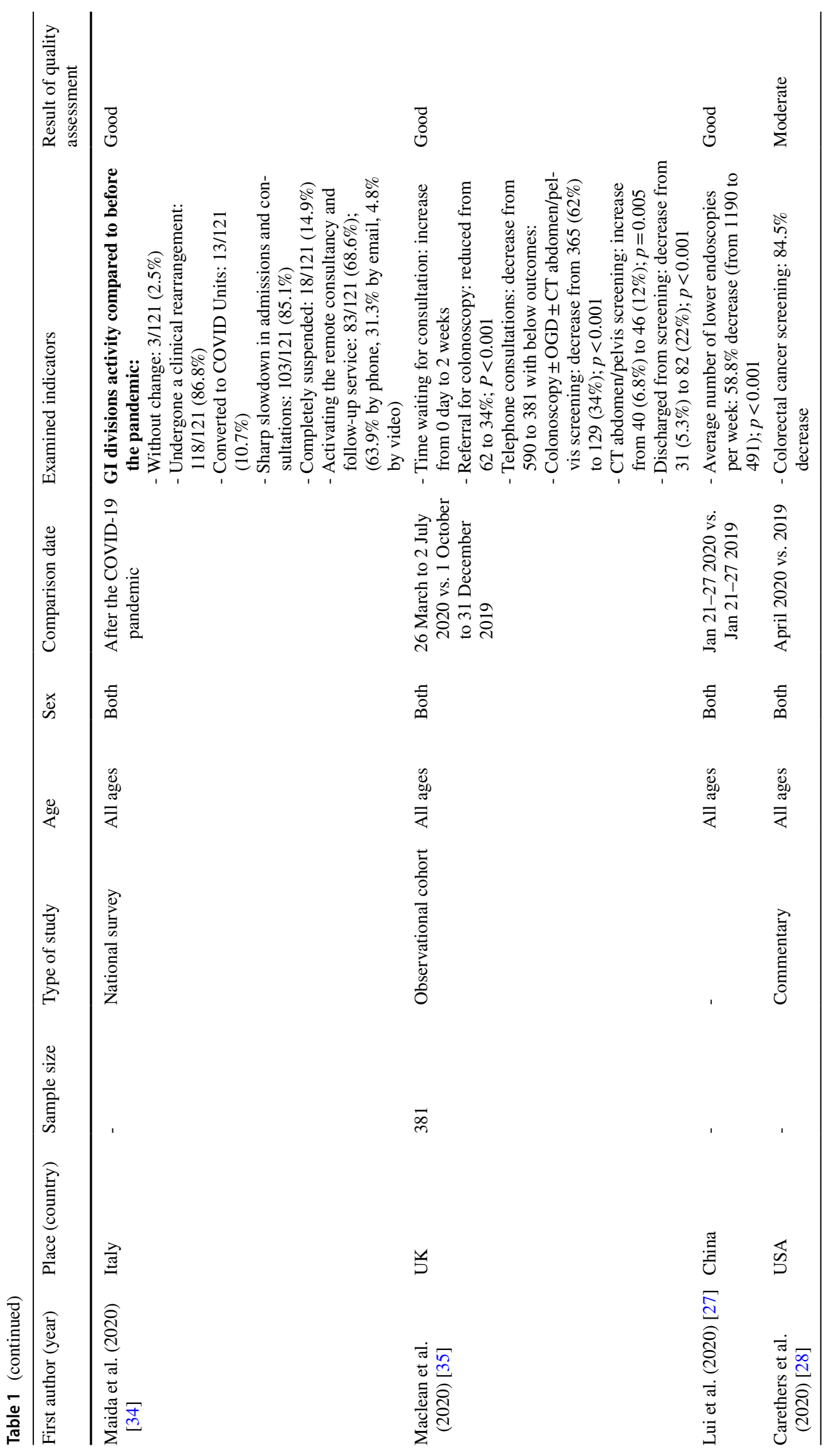


Fig. 1 Flow of information through the various phases of the systematic review

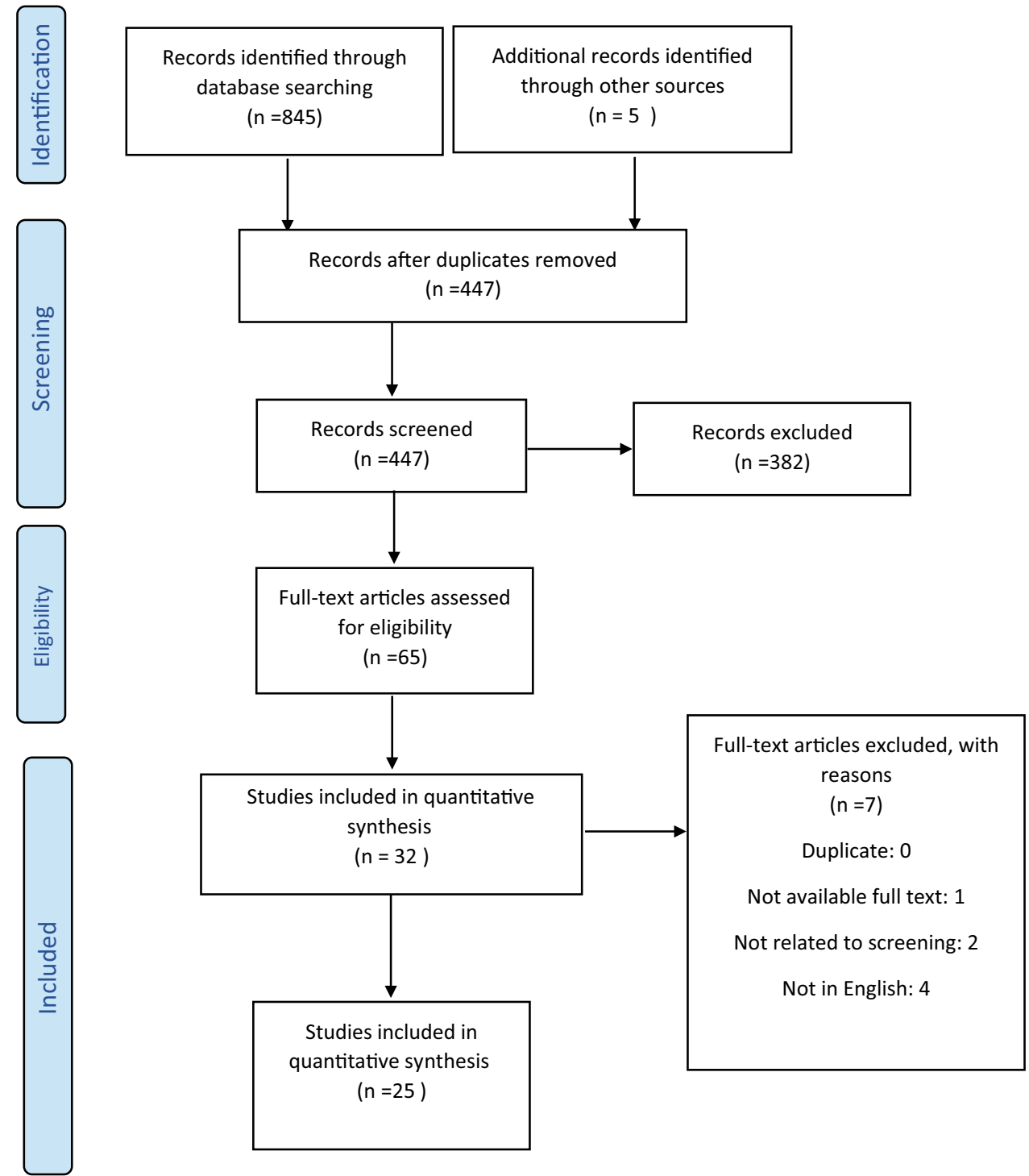

the two time periods before and after the constraints in 2019 and 2020 [18].

In a study by Lahat et al. in Israel, which compared endoscopy from January to March 2020 with the same period in 2019 and 2018, colonoscopy and sigmoidoscopy decreased by 52-57\% in 2020 (464 in 2020 compared to 955 and 1058 in 2018 and 2019) $(p<0.0001)$ [19].

A study by Kirac et al. in Croatia from August 1, 2019, to August 31, 2020, showed that the number of colonoscopy cases decreased from 50 in August 2019 to less than 9 in April 2020, but 3 months later return to normal condition [20].

Gurney et al. in their study found that New Zealand experienced a sharp decline in the number of gastrointestinal endoscopies during the National Corona Restriction Period (late March and April 2020); as the number of gastroscopic and colonoscopic cases in April 2019 decreased from approximately 5734 cases to less than 1426 cases in April 2020, and then, with a gradual increase until August 2020, it returned to the same number of before March [21].

In a study in the USA, Gorin et al. compared cancer screening between March 19 and May 9 in 2017, 2018, 2019, and 2020. They concluded that the number of colonoscopy cases has decreased from 1291 to 8 cases [22].

Al-Kuwari et al. conducted a study in Qatar, during the period of March to July 2019, and reported 5854 missing colonoscopies, and from April to July 2020, colorectal cancer screening services have decreased by $100 \%$ [23].

A study by Cheng et al. in Taiwan found that the screening uptake rate from winter 2019 to spring 2020 was $88.1 \%$, which was significantly lower than the previous 3 years (92.1\% from winter 2018 to Spring 2019, 91.2\% from winter 2017 to spring 2018, and $92.7 \%$ from winter 2016 to spring 2017) ( $P$ for trend <0.0001) [24]. 
London et al. in a UK study found that from January to April 2020, colorectal cancer screening decreased by $84.5 \%$ compared to the same period in 2019 [25].

A study by Mizuno et al. in Japan showed that with the release of COVID-19 in March 2020 in Kyoto, the number of outpatients and screening or diagnostic colonoscopy decreased (14.1\%) and continued for some time. After the cancelation of the emergency on May 21, 2020, the number increased again [26].

In a study by Lui et al. in Hong Kong, it was found that on January 21-27, 2020 (after the diagnosis of the first case of the coronary artery), compared to the same period in 2019 , the average of lower endoscopy decreased by $58.8 \%$ and reached from 1190 cases per week to 491 cases $(p<0.001)$ [27].

Carethers, J.M. et al. in the USA, by comparing screening tests for colon cancer in April 2020 and 2019, found that it had decreased by $84.5 \%$ in 2020 [28].

\section{Surveillance Colonoscopy}

A study by Wassie et al. comparing the number of colonoscopies in Australia in two similar timeframes (April-June) in 2019 and 2020 found that during the study period in 2020, the proportion of surveillance colonoscopy decreased. There were 597 cases of surveillance colonoscopy evaluated during the period, including $22.6 \%$ that recalled after 3-year care interval $(n=135)$ and $36.5 \%$ that recalled after a 5-year interval $(n=218)$ [12].

According to the fact that previous dates are based on the results of previous colonoscopy pathology for up to 5 years, the total number of such colonoscopies in $2019(n=306)$ was similar to $2020(n=291)$. Of these, the number of care colonoscopies that were not completed 3 months later that time increased from $52.9(162 / 306)$ in 2019 to $68.0 \%$ $(198 / 291)$ in $2020(P<0.001)$. The number of surveillance colonoscopies that were not completed within 6 months of the deadline increased from $19.3(59 / 306)$ in 2019 to $46.1 \%$ $(134 / 291)$ in $2020(P<0.001)$. During the COVID-19 pandemic, the percentage of care colonoscopies with a delay of 3 months was higher in April compared to May and June. The majority of the delay in colposcopies was observed in patients with an interval of 3- and 5-year follow-up reminder during and before the COVID-19 pandemic [12].

A letter has been sent to consider another colonoscopy for patients over the age of 75 . In 2020, the number of nonresponse cases was significantly higher $(16 / 31,51.6 \%)$ than that observed in $2019(10 / 39,25.6 \%)(P=0.03)$. However, for respondents, there is no difference in the reservation request ratio $(62.1 \%$ in 2019 and $73.3 \%$ in $2020, P=0.46)$ and the patient less involved to perform the colonoscopy with the physician; therefore, during the period of
COVID-19, they were excluded from the Southern Cooperative Program for the Prevention of Colorectal Cancer (SCOOP) [12].

A study by Tinmouth et al. in Canada that compared colonoscopy cases from March to June 2020 with the same time period in 2019 found that reduction in surveillance screening in patients who required surveillance colonoscopy due to a previous polypectomy or colorectal cancer or a previous adenoma (low-risk or high-risk adenoma) was 79\% [13].

Lantinga et al. who compared the two time periods before and after the constraints in 2019 and 2020 in the Netherlands, found that surveillance colonoscopy which was the most important cause of colonoscopy in 2019 (35.0\% (95\% CI: 33.9-36.1)) was decreased to $19.4 \%$ (95\% CI: 33.9-36.1) in $2020(P<0.001)[18]$.

\section{Prescribed Colonoscopy}

A study by Wassie et al. which compared the number of colonoscopies in Australia in two similar time periods (April-June) in 2019 and 2020 found that performed colonoscopies due to abnormal abdominal radiology increased in April $2020(P<0.05)$. There was no difference in the colonoscopies rate performed before and after the epidemic due to a IBD or FIT positive test result or suspicious symptoms $(P>0.05)$, except for a slight increase in the number of positive FIT tests in May during the pandemic period $(P=0.003)[12]$.

A study conducted by Tinmouth et al. in Canada and has compared the colonoscopy performing from March to June 2020 with the same period in 2019 found that screening reduction was $80 \%$ in patients who have been screened for a family history of colorectal cancer; in patients screened for moderate risk of colorectal cancer, was $81 \%$; and in those who had screening because of suspicious symptoms, was $56 \%$. However, the colonoscopy number due to the positive results of the fecal immunochemical test (FIT) and guaiac fecal occult blood test (gFOBT), increase $8 \%$ and reached from 4390 cases in 2019 to 4758 cases in 2020 during the period of study [13].

A study by Cheng et al. which was conducted in Taiwan was reported that the colonoscopy rate in people with positive FIT test and colonoscopy prescribed for them was $66.1 \%$ in the spring of 2020, which was significantly lower than the same period in the last 3 years $(70.2 \%$ in $2017,77.5 \%$ in 2018, and $75.4 \%$ in 2019) $(P$ for trend $=0.017)$ [24].

\section{Emergency Colonoscopy}

In a study by Wassie et al. that compared the number of colonoscopy cases in Australia in two similar time periods 
(April-June) in 2019 and 2020, they found that the emergency colonoscopy rate increased from 71.2 (828/1163) in 2019 to $78.2 \%(445 / 569)$ in 2020 , which was accompanied by a significant decrease in the number of unusual colonoscopies from 335 to 124 (63.0\% decrease, $P=0.002)$. Although the total number of unusual incomplete colonoscopies decreased significantly during the pandemic, it increased from 4 cases in April to 35 cases in May and reached $73 \%(n=85)$ of the average capacity in 2019 by June [12].

Shinkwin et al. in their study in the UK showed that emergency colonoscopies increased from 28.6 in 2018 and 2019 to $36.0 \%$ in $2020(p=0.03)$ [14].

\section{Non-invasive Methods}

A study by Myint et al. that was conducted in the USA found that in the period before COVID-19 (1/29/2020 to $3 / 17 / 2020)$, performing the FIT test $(154.0 \pm 25.2$ Per week) that was the most screening tests after a colonoscopy during the cessation period significantly decreased to $60.6 \pm 52.7$ $(p<0.02)$ but several weeks later the cessation period began to increase [16].

They also found that in the months following the restarting of screening, although the use of several non-invasive screening methods including FIT $(154 \pm 25.2$ to $162 \pm 55.2$; $p=0.54$ ) and fecal DNA (0 to $6.0 \pm 3.2$ per week; $p<0.01$ ) is equal to or even greater than the pre-pandemic, the use of FS and CTC remained low during the study period [16].

\section{Referrals to Screening Colonoscopy}

The study by Miller et al., which examined the effects of COVID-19 on the diagnosis of colorectal cancer and compared it from the period of April 1 to May 31 in 2020 with the same period from 2017 to 2019 in the UK, showed that overall colonoscopy referrals decreased by $43 \%$, from an average of 1071 in previous years to 609 in 2020; this reduction includes a $79 \%$ (324 to 69 ) reduction in emergency referrals and a 64\% (581 to 211) reduction in routine referrals. Referrals from urgent suspicion of cancer (USOC) showed a $40 \%$ (235 to 329) increase [29].

\section{Completion of Screenings that Needed to Be Reviewed}

A study by Wassie et al. in Australia compared the number of colonoscopies in two similar time frames (April-June) in 2019 and 2020 which revealed that in 2019, 45.5\% $(n=1164.530)$ of the colonoscopies needed further examination, which decreased to $32.0 \%(n=1869 / 569)$ in 2020 , and as a result, the overall number of colonoscopies which need further examination decreased by $65.7 \%(P<0.001)[12]$.

\section{Responding to Recalls}

A study by Wassie et al. in Australia by comparing the number of colonoscopies in two similar timeframes (April-June) in 2019 and 2020 found that the average time spent for responding to a colonoscopy call letter has been comparable in 2019 (21 days, IQR 12-48 days) and 2020 (18 days, IQR 11-41 days) $(p=0.23)$. In addition, compared to the same period in $2019(18 / 102,17.6 \%)$, no significant difference was observed in the number of cases of non-response in the COVID-19 period $(34 / 162,21.0 \%)(p=0.44)$ [12].

In a study conducted by D'Ovidio et al. in Italy, they compared colonoscopy data for the period of March 9 to May 4, 2020, with the same time period in 2019 and concluded that in the period of limitations, 60 out of 137 invited patients underwent endoscopy, while at the same time, in 2019, 238 cases (3.9 times higher) underwent colonoscopy [30].

A study by Cheng et al. conducted in Taiwan found that in 2020, there is a reprogramming rate (change of schedule time for colonoscopy) of $7.8 \%$ due to change in schedule time and $3.1 \%$ due to colonoscopy cancelation in the COVID-19 epidemic. The overall rate of rescheduling or cancelation was $10.9 \%$, which was significantly higher than that in the previous 3 years $(P$ for trend $=0.023)$. They also found that in the spring of $2020,50 \%$ of people refused to have a diagnostic colonoscopy for fear of developing COVID-19, which had never happened in previous years [24].

\section{Participating in Screening Program}

Koczkodaj et al. in their study in Poland found that screening coverage and participation rate in colorectal cancer colonoscopy screening decreased to $7.09 \%$ in July 2020 , although this rate was $13.16 \%$ in 2019 [31].

\section{Sales of Laboratory Materials Needed for Colonoscopy}

Meyer et al. in a study in France concluded that following the reduction in colonoscopy cases during the COVID-19 pandemic period, during the 8-week period of restrictions, the distribution of colonoscopy drugs between March 30 and April 12 decreased by $85.6 \%$ compared to the expected number. Overall, only 83,045 colonoscopy drugs were distributed during the restrictions, which is $181,826(68.6 \%)$ less than expected [32]. 
Four months after the removal of the restrictions, the number of colonoscopy preparations remained lower than expected, but gradually returned to the expected level, but finally was the 73,761 (13.9\%) lower than expected distributions. In general, during the 6-month period after the outbreak of the pandemic, 25,558 cases of colonoscopy preparations were distributed less than expected [32].

\section{Reducing the Capacity of Screening Centers}

Boyle et al. in their study in England and Wales found that according to the COVID-19 incidence rate, $77 \%$ of hospitals reduced their diagnostic colonoscopy activities to $0-10 \%$ of their normal capacity; $21 \%$ of hospitals continued with 11 to $70 \%$ of their normal capacity; and only $2 \%$ of hospitals with 71 to $100 \%$ of their normal capacity continued to perform diagnostic colonoscopy [33].

A study by Maida et al. in Italy, after examining the activity of the gastroenterology divisions in hospitals, found that only $2.5 \%$ of the hospitals continued to operate as before and $86.8 \%$ changed the way they provide their services; as a result, outpatient admissions and counseling fell sharply by $85.1 \%$, and $14.9 \%$ of hospitals suspended operations [34].

\section{Changing the Screening Method and Admission Process in the Screening Centers}

Maclean et al. after changing strategies in a regional referral center to manage the crisis of COVID-19 (using the FIT test instead of colonoscopy and telephone counseling instead of face-to-face counseling for patient triage) by evaluating data from patients with colorectal cancer for two periods of March 26 to July 2 in 2020 and October 1 to December 31 in 2019 in the UK, found that the waiting time for counseling has increased from 0 days to 2 weeks; subsequent referrals for colonoscopy decreased from 62 to $34 \%(P<0.001)$. Following reducing telephone counseling from 590 to 381 cases, the counseling results also showed changes as colonoscopy $\pm \mathrm{OGD} \pm \mathrm{CT}$ abdomen/pelvis decreased from 365 $(62 \%)$ cases in 2019 to $129(34 \%)$ in $2020(P$-value $<0.001)$; CT abdomen/pelvis increased from $40(6.8 \%)$ cases in 2019 to $46(12 \%)$ cases in $2020(P$-value $<0.005)$ and the number of discharges increased from 31 (5.3\%) cases in 2019 to 82 $(22 \%)$ in $2020(P$-value $<0.001)$ [35].

A study conducted by Maida et al. in Italy assessed the activity of the gastrointestinal department in hospitals and found that $68.6 \%$ of hospitals to compensate for this decrease due to the crisis of COVID-19 established consulting services and remote monitoring $(63.9 \%$ launched telephone counseling, $31.3 \%$ email, and $4.8 \%$ video counseling) [34].

\section{Discussion}

This study investigated the effect of the COVID-19 disease pandemic on colorectal cancer screening by systematic review and the final analysis was performed based on 25 articles related to the purpose of the study.

Despite the recommendation to performing CRC screening in many countries of the world [37] and after approving and starting the mass screening programs for colorectal cancer such as breast and cervical cancer in many countries [38], due to the COVID-19 pandemic in many countries, cancer screening programs have been discontinued since March 2020 and screening disrupted for an unknown period of time [2].

Colorectal cancer screening by invasive methods (colonoscopy, flexible sigmoidoscopy, and CT colonography (virtual colonoscopy)) and non-invasive methods (fecal immunochemical test (FIT)), guaiac-based fecal occult blood test (gFOBT), multi-targeted stool DNA mt-s DNA, and CT colonography (virtual colonoscopy) is performed [39]. However, it is used differently depending on the degree of accuracy, sensitivity, and level of access in each community [4], but the most common method is colonoscopy.

The impact of the COVID-19 crisis on different colorectal cancer screening methods varies according to the applied guidelines and restrictions. Most studies have reported a decrease in colonoscopy screening [12-35] so that in some cases, the number of colonoscopies has reached zero [12, 33]; however, in some studies, this reduction was temporary and returned to normal sometime after the cessation of limitations [16, 20,21,26], while in some others this reduction continued throughout the postCOVID-19 pandemic period [13, 14].

One study prescript colonoscopy, which is requested due to history and probability of infection, suspicious symptoms, or a positive screening test result other than colonoscopy, is also increased [12], however, decreased in other studies [13, 14, 24].

The use of emergency colonoscopy due to the patient's emergency (rupture or obstruction of the large intestine) has been shown to increase in several studies $[12,14]$; however, in a study by Miller et al. in the UK, findings showed 79\% decline (from 324 to 69) in emergency referrals [29].

Surveillance colonoscopy that is performed periodically for people at high risk for colorectal cancer due to age or previous screening results also shows a significant reduction compared to the pre-period of COVID-19 [12, $13,18]$. Furthermore, cancelation of appointments [24] or non-response to calls was increased [30].

One study that compared colonoscopy referrals before and after the crisis found that both emergency and routine 
referrals decreased, while referred cases to emergency diagnostics centers by general practitioners increased by $40 \%$ [29].

Patients who did not complete a colonoscopy also increased [12]. The use of flexible sigmoidoscopy has also been significantly reduced during this period $[15,17,19]$.

The use of non-invasive methods shows less reduction than invasive methods. The main non-invasive screening method used to screen colorectal cancer is the FIT test, which in a study in the USA temporarily showed a significant reduction [16] but after the resumption of activities showed an increase [16]. However, where it is used as an alternative to colonoscopy [35], its number has increased so that its positive result has become one of the main reasons for colonoscopy $[13,18]$. Other methods had not changed significantly [16].

It is generally estimated that due to the decline in care services between 2020 and 2023 in the crisis of COVID-19, approximately $1,179,642$ to $2,014,164$ cases of colorectal cancer screening, 8346 to 12,894 cases of colorectal cancer diagnosis, and 6113 to 9301 cases of early-stage diagnoses for this cancer will be missed [11].

Given that these screening programs are performed with the aim of identifying precancerous lesions and cancers in the early stages in order to remove the lesions before they progress to tumors and treat the cancers in the early stages [38], it is expected that delays in diagnosis due to COVID19 epidemic cause a significant increase in the number of preventable cancer deaths [40, 41]. A study in the UK has predicted that according to the 5-year survival of patients, there were 1445 (1392-1591) to 1563 (1534-1592) additional deaths for colon cancer due to the COVID-19 crisis, equal to from 15.3 to $16.6 \%$ increase [40].

In addition, another study predicts that if the COVID-19 pandemic disrupts colon cancer screening services, its longterm effects could lead to thousands of deaths from colorectal cancer; if this disruption lasts up to 12 months, it would lead to the deaths of more than 3968 people in Australia, 2366 in Canada, and 1360-1762 in the Netherlands [41].

Therefore, some countries have considered compensatory measures to reduce the damage caused by this disease on screening and early diagnosis of colorectal cancer; these include setting up "cold sites" (non-COVID-19 treatment center) [33], using the FIT test instead of colonoscopy and telephone counseling instead of face-to-face counseling for patient triage [35]; in a modeling study, increasing the use of FIT from 15 to $22 \%$ over a 3-year period to compensate for the decline in colonoscopy screening due to the COVID19 crisis could lead to 655,825 screening cases and 2715 diagnosis of colon cancer, $72 \%$ of which will be diagnosed in the early stages [11].

It was also shown that in the short term, the increase in the use of fecal immunochemical tests was associated with approximately 588,844 additional colorectal cancer screening cases and 2836 colorectal cancer diagnoses, of which 1953 (68.9\%) were early-stage diagnoses [11]. In general, the time required to compensate for damage caused by the COVID-19 pandemic varies depending on the proportion of people who redirecting from low-yield colonoscopies to fecal immunochemical test [13]. A study which was conducted in Canada found that without any changes in workflow, the time required to make up for the backlog caused by the COVID-19 crisis was estimated as 41 months; while, by directing $25 \%, 50 \%$, and $75 \%$ of the colonoscopy to the FIT, the compensation time is reduced to 28,22 , and 19 months, respectively. If the direction is not changed to FIT, hospitals will need to increase their colonoscopy capacity to $124 \%$, $134 \%$, and $145 \%$ at 28,22 , and 19 months, respectively, to compensate for the delay [13].

Overall, the results of several studies revealed that increased use of fecal immunochemistry (FIT) during COVID-19 epidemic disease is associated with increased participation in colorectal cancer screening and further detection of early colorectal cancer [11]. It is also estimated that follow-up of canceled or delayed screening appointments as soon as possible can reduce the additional colorectal cancer deaths caused by the COVID-19 epidemic to $0.2 \%[41]$.

\section{Conclusion}

The results of studies show that the reduction of colorectal cancer screening due to the COVID-19 pandemic is completely influenced by the restrictions that have been implemented in different periods of time following the high prevalence of corona disease in different populations. This zigzag effect is visible in populations where the corona outbreak has intensified and the restrictions have been applied more than once.

Following a decrease in the number of screening cases, emergency colonoscopies have increased, followed by a significant decrease in the number of screening methods required to diagnose a case of cancer (17). Therefore, in order to compensate for the decline and prevent the continuation of this trend in colorectal cancer screening, the following suggestions are recommended to governments and government agencies and the public.

Governments and government agencies should consider implementing the following:

- Increasing public awareness by broadcasting educational and practical programs in mass media (radio and television) about the risk of colorectal cancer, the consequences and outcomes of delaying referral to diagnostic centers if there are suspicious symptoms, and the benefits 
of timely referral as well as encourage participation in screening programs.

- Providing short and useful educational media content regarding the items considered, by experts and their widespread dissemination through cyberspace in order to increase awareness, change attitudes, and reduce the fear of getting COVID-19 infection.

- Reassure people about the low risk of COVID-19 infection when visiting diagnostic centers through the practical application of preventive health protocols.

- Creating campaigns to support colorectal cancer to increase public knowledge and awareness using existing capacities, including health workers, university students, NGOs, and volunteer groups.

- Replacing non-invasive methods such as FIT testing instead of invasive methods in colorectal cancer screening programs.

- Practical training the FIT test sampling at home to the general public by educational and laboratory specialists.

- Increase the capacity of screening centers at the time of removal of restrictions in order to compensate for the reduction during the time of restrictions.

- Setting up and strengthening not in-person queuing systems (telephone and internet) in order to reduce congestion in diagnostic centers.

- Establish or strengthen telemedicine to reduce the number of face-to-face visits for diagnostic centers and to assist patients who do not have the ability or access to diagnostic centers

- Implementation of free or low-cost screening programs (due to the adverse effects of Covid-19 disease on the economic situation of the people)

- Prompt recall of people who have canceled or delayed their previous appointments for fear of developing COVID-19 infection.

- Provide the necessary forces for screening centers or return the relocated staff to their place of work during the cessation of the COVID-19 epidemic, in order to maintain the capacity of screening centers.

Things that people should keep in mind are:

- Considering the symptoms of colorectal cancer seriously and refer to diagnostic centers in a timely manner.

- Strict observance of health instructions for the prevention of COVID-19 when referring to diagnostic centers.

- Participating in screening programs with the encouragement of family, friends, and acquaintances.

- Participating and launching non-governmental campaigns in order to inform about colorectal cancer to other human beings.
Author Contribution A.F. and H.S. conceived and designed research. H.S. and A.T. conducted the research. A.F. and S.H. contributed to data extraction. All authors wrote the manuscript. All authors read and approved the manuscript.

Availability of Data and Material NA

\section{Declarations}

Ethics Approval NA

Consent to Participate NA

Competing Interests The authors declare no competing interests.

\section{References}

1. Li Q, Guan X, Wu P, Wang X, Zhou L, Tong Y, et al. Early transmission dynamics in Wuhan, China, of novel coronavirus-infected pneumonia. N Engl J Med. 2020;382(13):1199-207.

2. Richards M, Anderson M, Carter P, Ebert BL, Mossialos E. The impact of the COVID-19 pandemic on cancer care. Nature Cancer. 2020;1(6):565-7.

3. Kaufman HW, Chen Z, Niles J, Fesko Y. Changes in the number of US patients with newly identified cancer before and during the coronavirus disease 2019 (COVID-19) pandemic. JAMA netw open. 2020;3(8):e2017267.

4. Zahedi A, Rafiemanesh H, Enayatrad M, Ghoncheh M, Salehiniya H. Incidence, Trends and Epidemiology of Cancers in North West of Iran. Asian Pac J Cancer Prev. 2015;16(16):7189-93.

5. Sung H, Ferlay J, Siegel RL, Laversanne M, Soerjomataram I, Jemal A, et al. Global Cancer Statistics 2020: GLOBOCAN estimates of incidence and mortality worldwide for 36 cancers in 185 countries. CA: Cancer J Clin. 2021;71(3):209-49.

6. McBain RK, Cantor JH, Jena AB, Pera MF, Bravata DM, Whaley $\mathrm{CM}$. Decline and rebound in routine cancer screening rates during the COVID-19 pandemic. J Gen Intern Med. 2021;36(6):1829-31.

7. Yong JH, Mainprize JG. The impact of episodic screening interruption: COVID-19 and population-based cancer screening in Canada. 2020:969141320974711.

8. De Vincentiis L, Carr RA, Mariani MP, Ferrara G. Cancer diagnostic rates during the 2020 'lockdown', due to COVID-19 pandemic, compared with the 2018-2019: an audit study from cellular pathology. 2021;74(3):187-9.

9. Kammar P, Chaturvedi A, Sivasanker M, de'Souza A, Engineer R, Ostwal $\mathrm{V}$, et al. Impact of delaying surgery after chemoradiation in rectal cancer: outcomes from a tertiary cancer centre in India. J Gastrointest Oncol. 2020;11(1):13-22.

10. Simunovic M, Rempel E, Thériault ME, Baxter NN, Virnig BA, Meropol NJ, et al. Influence of delays to nonemergent colon cancer surgery on operative mortality, disease-specific survival and overall survival. Canadian journal of surgery Journal canadien de chirurgie. 2009;52(4):E79-e86.

11. Issaka RB, Taylor P, Baxi A, Inadomi JM, Ramsey SD, Roth J. Model-based estimation of colorectal cancer screening and outcomes during the COVID-19 pandemic. JAMA netw open. 2021;4(4):e216454.

12. Wassie MM, Agaciak M, Cock C, Bampton P, Young GP. The impact of coronavirus disease 2019 on surveillance colonoscopies in South Australia. 2021;5(4):486-92. 
13. Tinmouth J, Dong S, Stogios C, Rabeneck L, Rey M, Dubé C. Estimating the backlog of colonoscopy due to coronavirus disease 2019 and comparing strategies to recover in Ontario. Canada Gastroenterology. 2021;160(4):1400-2.e1.

14. Shinkwin M, Silva L, Vogel I, Reeves N, Cornish J, Horwood $\mathrm{J}$, et al. COVID-19 and the emergency presentation of colorectal cancer. Colorectal Dis: the official journal of the Association of Coloproctology of Great Britain and Ireland. 2021.

15. Rutter MD, Brookes M, Lee TJ, Rogers P, Sharp L. Impact of the COVID-19 pandemic on UK endoscopic activity and cancer detection: A National Endoscopy Database Analysis. Gut. 2021;70(3):537-43.

16. Myint A, Roh L, Yang L, Connolly L, Esrailian E, May FP. Noninvasive colorectal cancer screening tests help close screening gaps during COVID-19 pandemic. JGH open : an open access $\mathbf{J}$ Gastroenterol Hepatol. 2021.

17. Longcroft-Wheaton G, Tolfree N, Gangi A, Beable R, Bhandari P. Data from a large Western centre exploring the impact of COVID-19 pandemic on endoscopy services and cancer diagnosis. 2021;12(3):193-9.

18. Lantinga MA, Theunissen F, Ter Borg PCJ, Bruno MJ, Ouwendijk RJT, Siersema PD. Impact of the COVID-19 pandemic on gastrointestinal endoscopy in the Netherlands: analysis of a prospective endoscopy database. Endoscopy. 2021;53(2):166-70.

19. Lahat A, Benjamin A. Changes in policy and endoscopic procedures during the 2019 coronavirus disease outbreak: A single center experience. Clinical Endoscopy. 2021;54(1):48-54.

20. Kirac I, Misir Z, Vorih V, Ćurt L, Śekerija M, Antoljak N. The impact of COVID-19 epidemiological restriction guidelines measures in a croatian tertiary colorectal cancer center. Libr Oncol. 2021;48(2-3):43-6.

21. Gurney JK, Millar E, Dunn A, Pirie R, Mako M, Manderson J, et al. The impact of the COVID-19 pandemic on cancer diagnosis and service access in New Zealand-a country pursuing COVID19 elimination. The Lancet Regional Health - Western Pacific. 2021;10.

22. Gorin SNS, Jimbo M, Heizelman R, Harmes KM, Harper DM. The future of cancer screening after COVID-19 may be at home. Cancer. 2021;127(4):498-503.

23. Al-Kuwari MG, Abdulmalik MA, Al-Mudahka HR, Bakri AH, Al-Baker WA, Abushaikha SS, et al. The impact of COVID-19 pandemic on the preventive services in Qatar. J Public Health Res. 2021;10(1):1910.

24. Cheng, S Y., Coldman A, Nadeau C, Iragorri N, Hilsden RJ, Brenner DR, et al. Impact of COVID-19 pandemic on fecal immunochemical test screening uptake and compliance to diagnostic colonoscopy. J Med Screen. 2020.

25. London JW, Braude P, Vilches-Moraga A, Hewitt J, Carter B, London JW, et al. Effects of the COVID-19 pandemic on cancerrelated patient encounters. Colorectal disease : the official journal of the Association of Coloproctology of Great Britain and Ireland. 2020;4:657-65.

26. Mizuno R, Ganeko R, Takeuchi G, Mimura K, Nakahara H, Hashimoto K, et al. The number of obstructive colorectal cancers in Japan has increased during the COVID-19 pandemic: a retrospective single-center cohort study. Expert Rev Mol Diagn. 2020;60:675-9.

27. Lui TKL, Leung K, Guo CG, Tsui VWM, Wu JT, Leung WK. Impacts of the coronavirus 2019 pandemic on gastrointestinal endoscopy volume and diagnosis of gastric and colorectal cancers: a population-based study. Gastroenterology. 2020;159(3):1164-6.e3.
28. Carethers JM, Sengupta R, Blakey R, Ribas A, D'Souza G. Disparities in cancer prevention in the COVID-19 era. Cancer Prev Res. 2020;13(11):893-6.

29. Miller J, Maeda Y. Short-term outcomes of a COVID-adapted triage pathway for colorectal cancer detection. Cancer causes \& control : CCC. 2021.

30. D’Ovidio V, D’Ovidio V, Lucidi C, Bruno G, Lisi D, Miglioresi L, et al. Impact of COVID-19 pandemic on colorectal cancer screening program. Br J Surg. 2021;20(1):e5-11.

31. Koczkodaj P, Sulkowska U, Kamiński MF, Didkowska J. SARS-CoV-2 as a new possible long-lasting determining factor impacting cancer death numbers. Based on the example of breast, colorectal and cervical cancer in Poland. Nowotwory. 2021;71(1):42-6.

32. Meyer A. Colonoscopy in France during the COVID-19 pandemic. Colorectal disease : the official journal of the Association of Coloproctology of Great Britain and Ireland. 2021;36(5):1073-5.

33. Boyle JM, Kuryba A, Blake HA, Aggarwal A, van der Meulen J, Walker K, et al. The impact of the first peak of the COVID-19 pandemic on colorectal cancer services in England and Wales: a national survey. Colorectal disease : the official journal of the Association of Coloproctology of Great Britain and Ireland. 2021.

34. Maida M, Sferrazza S, Savarino E, Ricciardiello L, Repici A, Morisco F, et al. Impact of the COVID-19 pandemic on gastroenterology divisions in Italy: a national survey. Digestive and liver disease : official journal of the Italian Society of Gastroenterology and the Italian Association for the Study of the Liver. 2020;52(8):808-15.

35. Maclean W, Limb C, Mackenzie P, Whyte MB, Benton SC, Rockall T, et al. Adoption of faecal immunochemical testing for 2-week-wait colorectal patients during the COVID-19 pandemic: an observational cohort study reporting a new service at a regional centre. Int J Colorectal Dis. 2020.

36. Morris EJA, Goldacre R, Spata E, Mafham M, Finan PJ, Shelton J, et al. Impact of the COVID-19 pandemic on the detection and management of colorectal cancer in England: a population-based study. The lancet Gastroenterology \& hepatology. 2021;6(3):199-208.

37. Schreuders EH, Ruco A, Rabeneck L, Schoen RE, Sung JJ, Young GP, et al. Colorectal cancer screening: a global overview of existing programmes. Gut. 2015;64(10):1637-49.

38. Gini A, Jansen EEL, Zielonke N, Meester RGS, Senore C, Anttila A, et al. Impact of colorectal cancer screening on cancer-specific mortality in Europe: a systematic review. Eur J Cancer. 2020;127:224-35.

39. Maida M, Macaluso FS, Ianiro G, Mangiola F, Sinagra E, Hold $\mathrm{G}$, et al. Screening of colorectal cancer: present and future. Expert Rev Anticancer Ther. 2017;17(12):1131-46.

40. Maringe C, Spicer J, Morris M, Purushotham A, Nolte E, Sullivan $\mathrm{R}$, et al. The impact of the COVID-19 pandemic on cancer deaths due to delays in diagnosis in England, UK: a national, populationbased, modelling study. Lancet Oncol. 2020;21(8):1023-34.

41. de Jonge L, Worthington J, van Wifferen F, Iragorri N, Peterse EFP, Lew JB, et al. Impact of the COVID-19 pandemic on faecal immunochemical test-based colorectal cancer screening programmes in Australia, Canada, and the Netherlands: a comparative modelling study. Communications biology. 2021;6(4):304-14.

Publisher's Note Springer Nature remains neutral with regard to jurisdictional claims in published maps and institutional affiliations. 